intraluminal tumor, bronchoscopy and frozen sections allowed us to guarantee a negative margin and still preserve lung function. Obviously, it was a big challenge to conduct the anastomosis of 3 tubes, including the left main bronchus, left upper bronchus, and left lower bronchus, in a complete VATS procedure. Such a task is best attempted by surgeons experienced in both advanced VATS and bronchoplasty to deal with such variant suture angles and long anastomotic length. Because the advantage of VATS lobectomy versus open surgery has been well established, ${ }^{2}$ however, we think that our operation will hold better functional benefits for this patient.

Finally, according to the experience of MD Anderson Cancer Center, ${ }^{3}$ adjuvant postoperative radiotherapy can improve the disease-specific survival for patients with tracheal or bronchial squamous cell cancer. Although there is no consensus on the role of adjuvant radiotherapy, we therefore believe that postoperative radiotherapy was also very important for this patient.

\section{References}

1. Mahtabifard A, Fuller CB, McKenna RJ Jr. Video-assisted thoracic surgery sleeve lobectomy: a case series. Ann Thorac Surg. 2008;85:S729-32.

2. McKenna RJ Jr, Houck W, Fuller CB. Video-assisted thoracic surgery lobectomy: experience with 1,100 cases. Ann Thorac Surg. 2006;81:421-5; discussion 425-6.

3. Webb BD, Walsh GL, Roberts DB, Sturgis EM. Primary tracheal malignant neoplasms: the University of Texas MD Anderson Cancer Center experience. J Am Coll Surg. 2006;202:237-46.

\title{
Unique case of total artificial cardiac support in failed Fontan circulation after cardiectomy: Is continuous flow better than pulsatile flow?
}

Christina J. VanderPluym, MD, Nee S. Khoo, MD, Ivan M. Rebeyka, MD, and Holger Buchholz, MD, Edmonton, Alberta, Canada

We report a case of total cardiectomy with implantation of a left ventricular assist device (VAD) for postcardiotomy Fontan circulation failure. This unique experience provides valuable insights into the physiologic consequences of pulsatile versus continuous flow (CF) VADs in the singleventricle circulation.

\section{CLINICAL SUMMARY}

A male infant with tricuspid atresia 1C underwent pulmonary artery banding shortly after birth, followed by a bidirectional cavopulmonary anastomosis at 6 months. At 2.8 years of age, he underwent an extracardiac fenestrated Fontan procedure and was extubated in the operating room. Excessive chest tube losses prompted reexploration 8 hours after surgery. Uncontrollable hemorrhage from an

From the Division of Cardiology and Cardiovascular Surgery, Department of Pediatrics, Stollery Children's Hospital, University of Alberta, Edmonton, Alberta, Canada.

Disclosures: Authors have nothing to disclose with regard to commercial support.

Received for publication Dec 14, 2012; revisions received Feb 6, 2013; accepted for publication Feb 13, 2013; available ahead of print March 20, 2013.

Address for reprints: Christina J. VanderPluym, MD, Division of Pediatric Cardiology, University of Alberta \& Stollery Childrens Hospitals, Edmonton, Alberta,

Canada (E-mail: christina.vanderpluym@ childrens.harvard.edu).

J Thorac Cardiovasc Surg 2013;145:e62-3

$0022-5223 / \$ 36.00$

Copyright (c) 2013 by The American Association for Thoracic Surgery

http://dx.doi.org/10.1016/j.jtcvs.2013.02.031 extensive myocardial hematoma was identified. The patient was placed on cardiopulmonary bypass; however, further measures to control bleeding from a myocardial dehiscence along the diaphragmatic surface of the heart were unsuccessful. The heart was completely excised along the atrioventricular groove while maintaining the Fontan pathway intact, and the ascending aorta was transected at the sinotubular junction with removal of all native valves. A 30-mm Gelweave graft (Terumo Cardiovascular Systems Corporation, Ann Arbor, Mich) was anastomosed to the left atrial cuff, forming a reservoir for pulmonary venous return and connected to a 9-mm Berlin Heart (BH) inflow cannula. A 16-mm Gore-Tex graft (W. L. Gore \& Associates, Flagstaff, Ariz) was sewn end-to-end to the ascending aorta, permitting placement of a 6-mm BH outflow cannula. A CentriMag CF pump (Thoratec Corporation, Pleasanton, Calif) was connected to the $\mathrm{BH}$ cannulas as an interim device during the wait for patient stabilization. The CentriMag resulted in stable flow, with a mean arterial pressure of 50 to $70 \mathrm{~mm} \mathrm{Hg}$ and a central venous pressure of 12 to $14 \mathrm{~mm} \mathrm{Hg}$. An $80-\mathrm{mL}$ saline tissue expander was placed within the empty pericardial sac to maintain space for future transplantation.

The patient was extubated, alert, and interactive on day 6 of support. At day 10, a mobile thrombus in the circuit necessitated pump change. In light of the patient's hemodynamic stability and improving end organ function, he the 


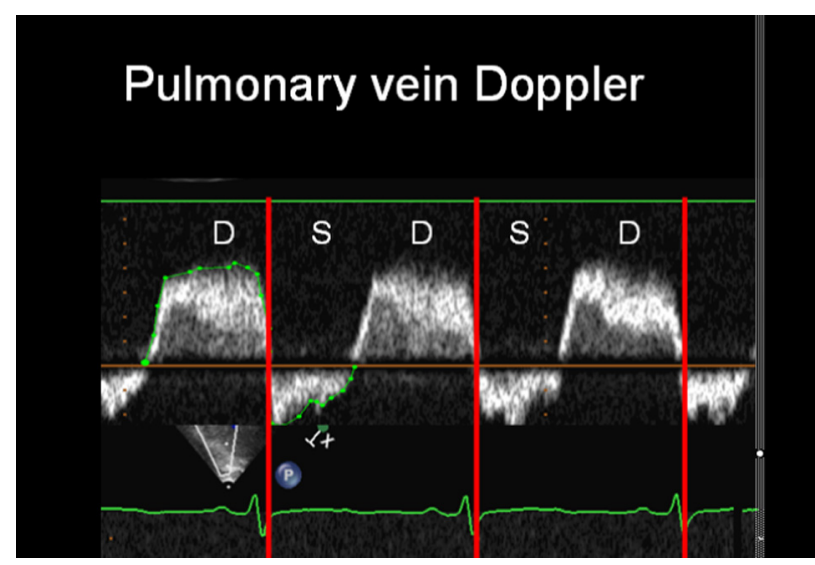

FIGURE 1. Pulmonary vein pulse wave Doppler with Berlin Heart EXCOR demonstrating reversal of flow into the pulmonary veins during pump systole $(S)$. $D$, Pump diastole.

pump was converted to a pulsatile flow BH EXCOR left ventricular assist device.

During the ensuing weeks, this patient acquired progressive pulmonary edema and vascular congestion refractory to diuresis. On day 28 of EXCOR support, he was reintubated for respiratory failure and subsequently required venovenous extracorporeal membrane oxygenation. Cardiac catheterization on day 37 of EXCOR support demonstrated an extensive plexus of decompressing venous collaterals and an elevated Fontan pressure of $24 \mathrm{~mm} \mathrm{Hg}$. Angiography demonstrated unobstructed pulmonary venous drainage to the Gelweave reservoir. Transcatheter coil occlusion of the collaterals transiently improved systemic oxygenation. Attempted optimization of EXCOR settings was subsequently performed under transesophageal echocardiographic guidance with interrogation of the pulmonary venous Doppler profiles. Figure 1 demonstrates the pulmonary venous Doppler profile with significant reversal of blood flow into the pulmonary veins during pump systole as a result of the absence of compliant native left atrial tissue and intact $\mathrm{BH}$ valves, despite a calculated cardiac index of $4.1 \mathrm{~L} /\left(\mathrm{min} \cdot \mathrm{m}^{2}\right)$.

To improve pulmonary and systemic venous congestion, VAD support was converted back to the CentriMag on day
44 of EXCOR support. The CentriMag CF pump resulted in an instantaneous decrease in Fontan pressures from 24 to 12 $\mathrm{mm} \mathrm{Hg}$ and clearing of the pulmonary congestion, allowing venovenous extracorporeal membrane oxygenation decannulation the next day. Unfortunately, on day 10 of CentriMag support, at a total of 54 days of VAD support, the patient had catastrophic mesenteric ischemia necessitating withdrawal of care.

\section{DISCUSSION}

VAD support with an intact Fontan pathway is possible after cardiectomy with currently available devices in children with careful consideration of device type, either continuous or pulsatile flow. Unique to this case was removal of the entire myocardial mass and replacement of the collecting chambers with noncompliant prosthetic material. This resulted in a physiologic situation similar to that of a failed Fontan circulation, with severe diastolic dysfunction and preserved systolic function. As such, use of a pulsatile-flow VAD was ineffective in unloading the pulmonary venous circulation, despite augmenting flow rates. The noncompliant Gelweave pulmonary venous reservoir attached to the fixed-volume EXCOR did not have sufficient capacity to ensure adequate decompression of the pulmonary veins, resulting in pulmonary edema, elevated Fontan pressures, and systemic venous hypertension. This was almost immediately rectified with conversion back to a continuous-flow device. Echocardiography proved useful in identifying this problem.

Successful use of pulsatile-flow VADs in failed Fontan circulations has been reported in the setting of systolic ventricular dysfunction. Fontan failure with elevated systemic venous pressures and diastolic dysfunction, however, may be better supported with CF devices. In this setting, we recommend support with a short-term CF pump (CentriMag), connected to $\mathrm{BH}$ cannulas for smaller patients, and an intracorporeal CF pump (HeartWare HVAD; International, Inc, Framingham, Mass) in larger patients $(>15 \mathrm{~kg})$. Further investigation is needed to determine optimal VAD support to suit the physiologic constraints of singleventricle circulation. 